\title{
A Descriptive Evaluation of Health Literacy and Determinants of COVID-19 Vaccine Acceptance among Patients with IgA Nephropathy with High Vaccine Uptake
}

\author{
Cynthia Ciwei Lim ${ }^{\mathrm{a}}$ Irene Y.J. Mok ${ }^{\mathrm{a}}$ Jun Jie Leeu ${ }^{\mathrm{b}}$ Zhong Hong Liew ${ }^{\mathrm{a}}$ \\ Hui Zhuan Tan ${ }^{a}$ Yok Mooi Chin ${ }^{a}$ Wei Ling Teng ${ }^{a}$ Fiona Yeo $^{c}$ Chieh Suai Tan ${ }^{a}$ \\ Jason C.J. Choo ${ }^{\mathrm{a}}$ \\ aDepartment of Renal Medicine, Singapore General Hospital, Singapore, Singapore; bee Kong Chian School \\ of Medicine, National Technological University, Singapore, Singapore; 'Pharmacy, Singapore General Hospital, \\ Singapore, Singapore
}

\section{Keywords}

Patient education $\cdot \mathrm{HLS}-\mathrm{EU}-\mathrm{Q} 47 \cdot$ Health literacy $\cdot$

Immunization

\begin{abstract}
Aims: Shared decision-making regarding COVID-19 vaccination in IgA nephropathy involves the ability to handle health information regarding potential benefits and risk of flare, but few studies have evaluated health literacy in the context of vaccination. We aimed to evaluate the health literacy and COVID-19 vaccination uptake and acceptance in IgA nephropathy. Methods: Single-center cross-sectional study of 126 consecutive patients with IgA nephropathy. Health literacy was assessed using the HLS-EU-47 questionnaire. Determinants of vaccine acceptance such as contextual influences, individual and group influences, and vaccine-specific issues were adapted from the World Health Organization framework. Results: Forty-eight patients (38.1\%) with IgAN nephropathy completed the survey between June and August 2021. The participants' median age was $40.5(31.6,52.8)$ years with median disease duration of $2.8(1.3,4.3)$ years. The
\end{abstract}

median general health literacy index was $31.74(29.88,35.82)$ with significantly greater difficulty in the competency of appraising health information and in the domain of disease prevention $(p<0.001)$. Forty-five patients $(93.8 \%)$ received at least one dose of COVID-19 vaccine between January and August 2021. Among the 3 unvaccinated patients, 2 intended to receive the vaccination while and 1 did not intend to get vaccinated. There was a high level of trust and belief that their government and healthcare providers had their best interests at heart and that the healthcare providers were honest about the vaccine's risk and benefits, although 31.2\% did not understand how the vaccine works and $22.9 \%$ believed that there were other ways to prevent infection. Most thought there was adequate safety information, were confident in the system for tracking adverse events and had no issues with access to the vaccine. Conclusion: Participants with IgA nephropathy had high health literacy scores and low vaccine hesitancy. The determinants for vaccine acceptance can potentially guide efforts to optimize vaccination coverage.

(C) 2022 The Author(s)

Published by S. Karger AG, Basel
Karger@karger.com www.karger.com/gdz

Karger $\stackrel{\text { ' }}{5}$

BOPEN ACCESS
(C) 2022 The Author(s)

Published by S. Karger AG, Basel

This is an Open Access article licensed under the Creative Commons Attribution-NonCommercial-4.0 International License (CC BY-NC) (http://www.karger.com/Services/OpenAccessLicense), applicable to the online version of the article only. Usage and distribution for commercial purposes requires written permission.
Correspondence to:

Cynthia Ciwei Lim, cynthia.lim.c.w@ singhealth.com.sg 


\section{Introduction}

Immunoglobulin A nephropathy is one of the most frequent primary glomerulonephritides diagnosed by kidney biopsies globally [1]. While the clinical presentation and course vary widely across geographical locations and ethnicities [2], IgA nephropathy has a predilection for progressive chronic kidney disease, such that endstage kidney disease can occur in up to $30 \%$ over $10-20$ years $[3,4]$. During the protracted disease clinical course, patients with $\operatorname{IgA}$ nephropathy may be at increased risk of infections due to clinical manifestations of nephrotic syndrome or impaired kidney function in chronic kidney disease and exposure to immunosuppressive therapy in selected patients $[4,5]$. During the ongoing COVID-19 pandemic, accrued data worldwide across the socio-geographic spectrum has convincingly shown that COVID-19 infection-related morbidity and mortality risks can be amplified in kidney disease and immunocompromised hosts $[6,7]$. Thus, leading expert opinion had empirically recommended COVID-19 vaccination for patients with immune-mediated kidney diseases [8], despite limited published data regarding vaccine efficacy in glomerulonephritides treated with immunosuppressants [9]. However, the lack of trial safety data in glomerulonephritides and reports of IgA nephropathy flares after vaccination that emerged in early 2021 [10-12] have led to calls for nephrologists to advocate COVID-19 vaccination but counsel patients regarding a small risk of relapsing disease for up to one month after the second dose and to monitor for symptoms suggestive of relapse [11].

The prerequisite for such shared decision-making is the ability to access, understand, evaluate, and apply healthcare-related information to make informed health decisions [13]. While data on health literacy among IgAN are scarce, our previous study of individuals with glomerulonephritides (conducted in mid-2020 before regulatory bodies approved the use of COVID-19 vaccines) noted that one of the areas with greatest perceived difficulty was related to appraising the need for vaccinations [14]. In particular, there is a real threat of misinformation about COVID-19 vaccines following an unprecedented manner of accelerated vaccine development and testing in 2020 then near-global exhortation to vaccinate immediately after vaccines became available [15]. While early studies among healthcare workers and the general public noted vaccine hesitancy in up to $70 \%$ even among high-income countries [16], there is little information regarding COVID-19 vaccine acceptance in IgA nephropathy or immune-mediated disease $[16,17]$. The extensive media coverage of the available vaccines over the past year may have altered the health literacy and acceptance of COVID-19 vaccination, which appears to be here to stay for the medium- to long-term since health authorities are now advising booster vaccinations for immunocompromised hosts [18]. Thus, there is a need to assess the prevalence of vaccine uptake and identify factors associated with vaccine hesitancy that can be addressed to improve protection for at-risk patients with IgA nephropathy. We thus aimed to evaluate the health literacy and factors associated with COVID-19 vaccination uptake and acceptance among patients with IgA nephropathy.

\section{Methods}

We performed a single-center cross-sectional study of 171 consecutive patients with biopsy-confirmed IgA nephropathy diagnosed between December 2015 and March 2021 at the Singapore General Hospital. After excluding those who had died $(n=8)$, received transplant $(n=5)$, on dialysis $(n=18)$, and not on active follow-up $(n=$ 14), we invited 126 patients to participate in either the telephone or in-person survey. The interviewer-administered questionnaire was conducted in English over the telephone by a single trained study member to minimize interviewer variability, while the self-administered survey (also in English) was offered in the clinic if the patient came for a routine nephrology consult. English was chosen since our pilot study noted that English was the most frequently used language in communicating with healthcare personnel and participants preferred the English version [14]. To reduce differences in the understanding of the questionnaire, patients had the opportunity to clarify any uncertainty about the survey, regardless of the modality. The following information was obtained using a standardized survey form: sociodemographic data such as age, gender, ethnicity, highest education attained, occupation personal gross monthly income (less than $\$ 4,000$ vs. $\geq \$ 4,000$ ), housing type (public vs. private), language spoken at home (English, Mandarin, Malay, Tamil, others) and frequency of visits to healthcare institutions such as clinic or hospital in the past 6 months. Health literacy was assessed using the HLS-EUQ47 questionnaire, a 47-item survey with each item rated on a 4-point Likert scale for perceived level of difficulty in the competencies of accessing, understanding, evaluating, and applying healthcare-related information in the domains of healthcare, disease prevention, and health promotion $[15,17]$. It had good construct validity, item-scale convergent validity, internal consistency, and no floor or ceiling effect [17]. The survey also evaluated the determinants of vaccine acceptance such as contextual influences, individual and group influences, and vaccine-specific issues as recommended by the World Health Organization framework (online suppl. Table S1; see www.karger.com/doi/10.1159/000522158 for all online suppl. material) [19]. Vaccine uptake (defined as received 1 or more vaccine doses), comorbid conditions, and medication history were obtained from the electronic medical records. Ethics review was not required for this service evaluation study as determined by the SingHealth Centralized Institutional Review Board (2021/2356) since participants were not subjected to additional risks or burdens beyond usual clinical practice. 


\section{Statistical Analysis}

Statistical analysis was performed using IBM SPSS Statistics 26 (IBM Corp., Armonk, NY, USA). Categorical variables were presented as proportions and continuous variables summarized as medians with interquartile ranges (25th percentile, 75th percentile). The health literacy index for each patient was standardized based on a formula: index $=($ mean score -1$) *(50 / 3)$, where mean score was the mean of all participating items that each patient answered, 50 was the chosen maximum value and 3 was the range of possible mean scores $[20,21]$. Thus, a health literacy index value was obtained where 0 represented the lowest and 50 represented the highest health literacy possible. To assess participants' ease with the competencies of accessing, understanding, appraising, and applying health information in the domains of healthcare, disease prevention and health promotion (online suppl. Table S2), the item mean scores (sum score/number of items with response) was calculated [22], then compared using the Friedman's test. The associations between health literacy scores and patient characteristics were evaluated using the Mann-Whitney $U$ test. All analyses were two-tailed, and $p$ values $<0.05$ were considered statistically significant.

\section{Results}

Forty-eight patients (38.1\%) with IgAN nephropathy completed the survey between June and August 2021: 40 by the interviewer-administered survey over telephone and 8 with the self-administered survey when they attended nephrology clinics. Among the nonresponders, 26 (20.6\%) rejected the survey due to language barrier, 12 (9.5\%) were not interested and the remaining were uncontactable despite repeated calling a week apart. Comparing responders and nonresponders (online suppl. Table S3), responders were significantly younger at the time of diagnosis but were not significantly different in gender, presence of comorbidities such as diabetes, hypertension, hyperlipidemia, clinical presentation, and uptake of COVID-19 vaccination $(93.8 \%$ vs. $80.8 \%$, respectively, $p=$ $0.20)$.

At the time of the study, the participants' median age was $40.5(31.6,52.8)$ years with median disease duration of $2.8(1.3,4.3)$ years. Among the multiethnic cohort (41 Chinese, 2 Indians, 3 Malays, and 2 other ethnicities), English was more frequently spoken at home (43.8\%) than Mandarin (41.7\%), Malay (6.3\%), Tamil $(2.1 \%)$, or other languages $(6.3 \%)$. The majority had completed at least secondary school education; only 1 participant did not have any formal education and 3 had primary school education. The median general health literacy index was $31.74(29.88,35.82)$. The item mean scores were 2.85 (2.62, 3.08), 3.09 (3.00, 3.42), $2.75(2.60,3.06)$, and $3.00(2.82,3.18)$ for the competen- cies of accessing, understanding, appraising, and applying healthcare-related information, respectively. The participants had significantly greater difficulty in appraising health information $(p<0.001)$. Ease with health information were higher in the domains of healthcare and health promotion but significantly lower in the domain of disease prevention (item mean score $3.00[2.86,3.22], 2.92[2.75,3.06]$, and $2.77[2.69,3.00]$, respectively; $p<0.001)$. Table 1 showed that younger participants $<50$ years old and those who spoke English at home had significantly higher general health literacy and item mean scores in the domains of healthcare and disease prevention. Younger age was also associated with higher scores for understanding health information. Participants who spoke English at home also had higher scores for accessing and applying health information. With regard to vaccination-specific items within the HLS-EU-47 questionnaire, most participants found it was fairly or very easy to find information on vaccinations (item number $19,85.4 \%$ ) and understand the need for vaccinations (item number $22,100 \%$ ) but half found judging which vaccines were needed (item number $26,50.0 \%)$ to be fairly or very difficult.

Forty-five patients $(93.8 \%)$ had received at least one dose of COVID-19 vaccine between January and August 2021. Among the remaining 3 unvaccinated patients, 2 intended to receive the vaccination while 1 did not intend to get vaccinated. All participants surveyed had heard about COVID-19 vaccines. Their information sources included the Internet or social media (89.6\%), family and friends (56.3\%), and healthcare providers (41.7\%). The determinants of COVID-19 vaccine acceptance were shown in Table 2. There was a high level of trust and belief that their government and healthcare providers had their best interests at heart and that the healthcare providers were honest about the vaccine's risk and benefits. Almost all were satisfied in their healthcare providers' answers to their queries regarding the vaccine. Most participants (87.2\%) thought the vaccine would strengthen the immune system although $31.3 \%$ did not understand how the vaccine works and $22.9 \%$ believed that there were other ways to prevent infection. While few participants had or knew others who had serious reactions to vaccines, the majority would be less willing to consider future vaccines if they did (75.6\%) or if they knew others who had serious reactions to vaccines $(68.9 \%)$. Regarding vaccine-specific issues, most participants thought there was adequate safety information, were confident in the system for tracking adverse events, and had no issues with access to the vaccine. 


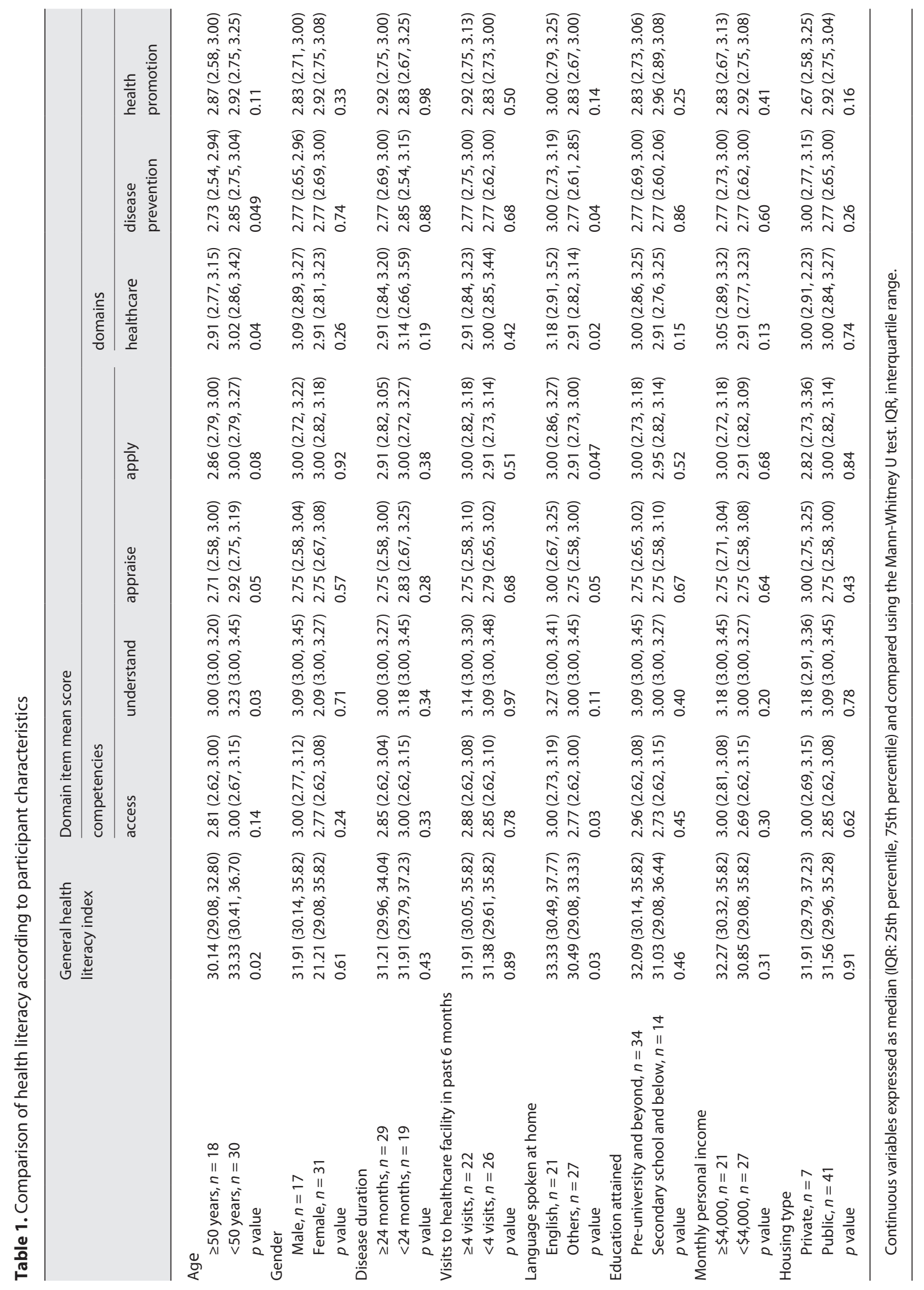


Table 2. Determinants of COVID-19 vaccine acceptance

Response, $N$ Yes, $N(\%)$

Contextual influences

What are the information sources you turn to for information for this vaccine?

a. Family and friends

b. Healthcare provider

c. Internet or social media

Will you avoid this vaccine because of religious or cultural reasons?

Do you trust that your government is making decisions in your best interest with respect to the vaccine provided?

Individual and group influences

Have you ever had a serious reaction to any vaccine?

If yes, will the experience make you less willing to consider this vaccine?

Do you know anyone who had a serious reaction to any vaccine?

If yes, will the experience make you less willing to consider future vaccination?

Have you ever had serious infection because you were not vaccinated?

Do you know anyone who had a serious infection because they were not vaccinated?

Do you believe that there are other better ways to prevent infection than with this vaccine?

Do you think the vaccine strengthens the immune system?

Do you understand how the vaccine works?

Do you feel that your medical conditions (including the medications you are taking) influence your decision to be

vaccinated?

Do you feel confused about the number or schedule of the vaccine?

Are you satisfied with your healthcare provider's answers to your questions related to this vaccine?

Do you trust your healthcare provider to honestly tell you about the risks and benefits of the vaccine?

Do you believe your healthcare provider has your best health interests at heart?

Vaccine-specific issues

Do you think there is adequate safety information?

As far as you know, are side effects or adverse reactions are being tracked?

How confident are you in the system for tracking adverse reactions or side effects?

Is access to this vaccination easy?

Is the location for this vaccination convenient?

Do you feel confident the vaccine supply is available when you need it?

$\begin{array}{ll}48 & 27(56.3) \\ 48 & 20(41.7) \\ 48 & 43(89.6) \\ 48 & 1(2.1) \\ 48 & 47(97.9) \\ & \\ 48 & 3(6.3) \\ 48 & 34(75.6) \\ 48 & 6(12.8) \\ 45 & 31(68.9) \\ 48 & 2(4.2) \\ 48 & 0 \\ 48 & 11(22.9) \\ 47 & 41(87.2) \\ 48 & 33(68.8) \\ 48 & 36(75.0) \\ 48 & 3(6.3) \\ 48 & 47(97.9) \\ 48 & 48(100) \\ 48 & 48(100) \\ & \\ 48 & 42(87.5) \\ 48 & 45(93.8) \\ 48 & 43(89.6) \\ 48 & 46(95.8) \\ 48 & 48(100) \\ 48 & 48(100) \\ & \end{array}$

\section{Discussion}

This cross-sectional study of 48 patients with IgA nephropathy with generally high literacy scores according to the HLS-EU-47 questionnaire noted a very low vaccine hesitancy rate $(2.1 \%)$, with most patients $(93.8 \%)$ having received at least one dose of COVID-19 vaccine over 8 months since the vaccines became available in the country. This was reflective of the national vaccine uptake with $93 \%$ of those $40-59$ years old having received at least one dose of the vaccine [23].

While the general health literacy was high, almost half of the cohort perceived difficulty with judging which vaccinations are needed and a-third did not understand how the COVID-19 vaccine worked. The ability to deal with health information in the domain of disease prevention, which would include vaccinations, was also significantly lower compared to the other 2 domains of healthcare and health promotion. Thus, other vaccine determinants may have contributed to the high vaccination rate observed, such as the high level of trust in their healthcare providers and government in relation to the COVID-19 vaccine. The Elderman Trust Barometer that measured trust in NGOs, business, government, and media (where trust level index $<50$ indicated distrust, 50-59 indicated neutral and score $>60$ indicated trust) found that Singaporeans' trust in the government increased by 6 points from 62 to 68 amidst the pandemic from 2020 to 2021 [24]. In addition, COVID-19 vaccinations were offered free-of-charge and at multiple sites to facilitate access to the vaccine. We were unable to analyze for factors associated with vaccine uptake or acceptance since only 1 responder did not intend to get vaccinated. However, in study cohorts dominated by the general public and healthcare workers, COVID-19 vaccine hesitancy was associated with younger age, female gender, lower education level, absence of chronic medical conditions and beliefs that the vaccine was not safe or effective [16]. As 
booster vaccinations are increasingly advocated for patients with kidney disease and immunocompromised hosts, including use of immunosuppressive therapy [18], strategies to optimize vaccination coverage among patients can focus on building upon the trust in their physicians' advice, addressing the concerns identified herein and improving vaccine-related health literacy by ensuring that health information is available in their mother tongues and using different communication mediums to reach out to older patients.

This study has some limitations. By including only patients on active follow-up and excluding patients who were lost to follow up, vaccination uptake and acceptance may be higher due to physicians advocating vaccination. In addition, selection bias may also result due to the use of English language for the survey. Since responders were younger than nonresponders and younger age was associated with higher health literacy, the general health literacy of the patients with IgA nephropathy is likely to be lower. Patients may also reject to participate due to negative views that they were unwilling to share [25]. While it was possible that voluntary participation may result in selection bias if nonresponders rejected the study because they had vaccine hesitancy, we found that vaccine uptake was not different between nonresponders and responders. The HLS-EU-47 questionnaire was intended to be self-administered but we adapted its administration to a telephone interview due to a move toward teleconsultations during the pandemic with reduced in-person clinic visits, while electronic mail or online self-administered surveys may result in even lower response rates since our patients with glomerulonephritis are increasingly older and some may be less adept using these technology [26]. Although few socioeconomic status measures differentiated health literacy in this cohort, this may be due to the predominantly middle-class nature of the cohort with few patients in the extremities of the socioeconomic status spectrum, thus limiting the ability to detect significant associations with health literacy.
In conclusion, this cohort of participants with IgA nephropathy had high health literacy and low vaccine hesitancy. The determinants for vaccine acceptance can potentially guide efforts to optimize vaccination coverage.

\section{Acknowledgments}

The study team thanks the Department of Renal Medicine, Singapore General Hospital, for their support for this project.

\section{Statement of Ethics}

Ethics review and written informed consent were not required for this service evaluation project as determined by the SingHealth Centralized Institutional Review Board (2021/2356) since participants were not subjected to additional risks or burdens beyond usual clinical practice. This study was conducted in accordance with the Declaration of Helsinki.

\section{Conflict of Interest Statement}

No relevant conflict of interest for all authors.

\section{Funding Sources}

No funding was received for this study.

\section{Author Contributions}

C.C.L. conceptualized the study and wrote the first draft; C.C.L., J.C.J.C., I.Y.J.M., and H.Z.T. recruited participants, J.J.L., Z.H.L., Y.M.C., W.L.T., and C.C.L. performed data entry and analysis; all the authors reviewed and approved the manuscript.

\section{Data Availability Statement}

Dataset is available upon reasonable request to the study team, subject to institutional approval and data sharing agreement.

\section{References}

1 Woo KT, Chan CM, Lim C, Choo J, Chin YM, Teng EWL, et al. A global evolutionary trend of the frequency of primary glomerulonephritis over the past four decades. Kidney Dis. 2019;5:247-58

2 Schena FP, Nistor I. Epidemiology of IgA nephropathy: a global perspective. Semin Nephrol. 2018;38:435-42.
3 Woo KT, Lim CC, Foo MW, Loh HL, Jin AZ, Chin YM, et al. 30-year follow-up study of IgA nephritis in a Southeast Asian population: an evaluation of the Oxford histological classification. Clin Nephrol. 2016;86(2016):270-8.

4 Barbour SJ, Canney M, Coppo R, Zhang H, Liu ZH, Suzuki Y, et al. Improving treatment decisions using personalized risk assessment from the International IgA nephropathy prediction tool. Kidney Int. 2020;98:1009-19.
5 Qian G, Zhang X, Xu W, Zou H, Li Y. Efficacy and safety of glucocorticoids for patients with IgA nephropathy: a meta-analysis. Int Urol Nephrol. 2019;51:859-68.

6 Windpessl M, Bruchfeld A, Anders HJ, Kramer H, Waldman M, Renia L, et al. COVID-19 vaccines and kidney disease. Nat Rev Nephrol. 2021;17:291-3. 
7 Fung M, Babik JM. COVID-19 in immunocompromised hosts: what we know so far. Clin Infect Dis. 2021;72(2):340-50.

8 Kronbichler A, Anders HJ, Fernandez-Juárez GM, Floege J, Goumenos D, Segelmark M, et al. Recommendations for the use of COVID-19 vaccines in patients with immunemediated kidney diseases. Nephrol Dial Transplant. 2021;36:1160-8.

9 Yeaman MR. Immunosuppression in glomerular diseases: implications for SARS-CoV-2 vaccines and COVID-19. Glomerular Dis. 2021;1(4):277-93.

10 Lim CC, Choo J, Tan CS. COVID-19 vaccination in immunoglobulin A nephropathy. Am J Kidney Dis. 2021;78(4):617.

11 Bomback AS, Kudose S, D’Agati VD. De novo and relapsing glomerular diseases after $\mathrm{CO}$ VID-19 vaccination: what do we know so far? Am J Kidney Dis. 2021;78(4):477-80.

12 Tan HZ, Tan RY, Choo JCJ, Lim CC, Tan CS, Loh AHL, et al. Is COVID-19 vaccination unmasking glomerulonephritis? Kidney Int. 2021;100(2):469-71.

13 Sorensen K, Van den Broucke S, Fullam J, Doyle G, Pelikan J, Slonska Z, et al. Health literacy and public health: a systematic review and integration of definitions and models. BMC Public Health. 2012;12:80.
14 Lim CC, Mok IYJ, Tan HZ, Tan C, Yeo F, Choo JCJ. Health literacy in glomerulonephritis and renal vasculitis attending nephrology clinics. Glomerular Dis. 2021;1(3):12934.

15 Montagni I, Ouazzani-Touhami K, Mebarki A, Texier N, Schück S, Tzourio C, et al. Acceptance of a Covid-19 vaccine is associated with ability to detect fake news and health literacy. J Public Health. 2021;43(4):695-702.

16 Aw J, Seng JJB, Seah SSY, Low LL. COVID-19 vaccine hesitancy-A scoping review of literature in high-income countries. Vaccines. 2021;9(8):900.

17 Sim J, Lim CC. Coronavirus disease 2019 (COVID-19) vaccination in systemic lupus erythematosus and anti-neutrophil cytoplasmic antibody-associated vasculitis. Clin Rheumatol. 2021;40(6):2517-8.

18 Wise J. Covid-19: UK will offer third vaccine dose to severely immunosuppressed people British Medical Journal Publishing Group; 2021.

19 MacDonald NE. Vaccine hesitancy: definition, scope and determinants. Vaccine. 2015; 33:4161-4.

20 Sorensen K, Pelikan JM, Rothlin F, Ganahl K, Slonska Z, Doyle G, et al. Health literacy in Europe: comparative results of the European health literacy survey (HLS-EU). Eur J Public Health. 2015;25:1053-8.
21 Duong TV, Aringazina A, Baisunova G, Nurjanah D, Pham TV, Pham KM, et al. Measuring health literacy in Asia: validation of the HLS-EU-Q47 survey tool in six Asian countries. J Epidemiol. 2017;27:80-6.

22 van der Heide I, Rademakers J, Schipper M, Droomers M, Sørensen K, Uiters E. Health literacy of Dutch adults: a cross sectional survey. BMC public health. 2013;13:179.

23 Singapore $\mathrm{MoH}$. Update on local COVID-19 situation and vaccination progress (30 August 2021) [Internet] 29th September 2021]. Available from: https://www.moh.gov.sg/newshighlights/details/update-on-local-covid19-situation-and-vaccination-progress-(30august-2021)

24 Edelman. 21st Annual Edelman Trust Barometer. [Internet], 2021 [cited 2021 October 25th ]. Available from: https://www.edelman. $\mathrm{com} / \mathrm{sites} / \mathrm{g} /$ files/aatuss191/files/2021-03/ 2021\%20Edelman\%20Trust\%20Barometer Singapore\%20Country\%20Report_1.pdf.

25 Booker QS, Austin JD, Balasubramanian BA. Survey strategies to increase participant response rates in primary care research studies. Fam Pract. 2021;38(5):699-702.

26 Lim CC, Huang H, Chin YM, Teng WL, Woo KT. Kidney biopsy in the elderly: safety and strategies to prevent uremic bleeding. $\mathrm{Ne}-$ phrology. 2019;24:876. 